\title{
Intelligent Watermarking with Multi-Objective Population Based Incremental Learning
}

\author{
Bassem S. Rabil, Robert Sabourin and Eric Granger \\ Laboratoire d'Imagerie, de Vision, et d'Intelligence Artificielle \\ École de Technologie Superiéure, Université de Quebec \\ 1100, rue Notre-Dame Ouest, Montreal, Canada, H3C 1K3 \\ bguendy@livia.etsmtl.ca, Robert.Sabourin@etsmtl.ca and Eric.Granger@etsmtl.ca
}

\begin{abstract}
Intelligent watermarking techniques use nonconventional methods like Evolutionary Computation techniques to satisfy the trade-off between integrity and authenticity of digitized documents. In this paper, we propose a multi-objective Population Based Incremental Learning module for an intelligent watermarking system for grayscale images to optimize embedding watermarks that satisfy the trade-off between quality and robustness. The multi-objective formulation provides set of non-dominated solutions rather than single solution, which allows tuning the quality and robustness for several attacks, without the need for an expensive re-optimization process due to changing the priority of different objectives after the optimization process. Different formulations for the optimization problem were investigated to achieve best fitness for different objectives. The best fitness achieved for all objectives are compared for different formulations. Simulation results indicate better fitness for different objectives with multi-objective formulation, and faster convergence using incremental learning techniques.
\end{abstract}

Keywords-intelligent watermarking; multi-objective; incremental learning

\section{INTRODUCTION}

With rapid development of information technology, growing number of digital documents are transmitted and exchanged via the Internet. It has created an environment where digital information is easily distributed, duplicated and modified, leading to the need for effective copyright protection techniques. In addition, the digitization of documents allows the rapid dissemination of data in distributed systems and post processing on computerized resources. Out of the main challenges consists in assuring the integrity, authenticity, and confidentiality of these digitized documents images. Various digital watermarking schemes have been developed to address these issues.

Digital watermarking is deployed in many domains to assure the integrity and authenticity of the original signal via fragile and robust watermarking respectively. Digital watermarking is the process of embedding information into a digital signal. The signal may be audio, pictures or video. If the signal is copied, then the information is also carried in the copy. Biometrics and cryptography can be integrated with watermarking framework to assure confidentiality. A fragile watermark is a type of watermark to ensure integrity, but it is broken if the watermarked image is manipulated or altered, while the robust watermark ensures authenticity and can be extracted after manipulating the watermarked image. Semi-fragile watermark is satisfying a trade-off between both quality and robustness.

Most digital watermarking techniques proposed for grayscale images use different transform domains to embed a watermark that minimizes the visual impact, and to deal with the uncorrelated coefficients in the transform domain. The most commonly used transform domains are Discrete Cosine Transform (DCT) and Discrete Wavelet Transform (DWT). Using DCT transform, the host image is divided into small blocks of pixels ( $8 \times 8$ pixels), transformed to frequency domain, and watermark bits are distributed among these blocks by changing frequency bands coefficients of these blocks according to the value of the watermark bit to be embedded. Some authors have proposed embedding multiple watermarks to address authenticity and integrity separately. Using two different watermarks exploits the fact that there are some frequency band coefficient ranges which have better robustness when embedding watermark bits, and other ranges of minimal visual impact of embedding the watermark.

Haouzia and Noumeir [4] pointed out the need for watermarking system that can be tuned by the operator to increase the robustness against certain attacks according to the type of digitized documents to be watermarked. Using intelligent watermarking techniques may translate to a complex high dimension optimization problem to find the optimal bands for embedding the watermark. The dimension of the optimization is large because the metrics of the objectives to be optimized, which are quality and robustness, are calculated for the whole image while being dependent on the embedding bands in each and every image block. Thus, all embedding bands must be represented among the individuals of the optimization population. To converge for high dimensional problem as in intelligent watermarking in less iterations, we recommended incremental learning mechanisms to account for all previous generations experience to reach the convergence.

In this paper, it is proposed to formalize the intelligent watermarking as Multi-Objective Optimization Problem (MOOP) to optimize simultaneously quality and robustness against different attacks using a multi-objective PBIL algorithm, producing multiple non-dominated solutions (Pareto front). This gives a system operator multiple optimal solutions to choose among to favor the robustness against certain attacks over other attacks. Different problem formulations have been 
evaluated in this paper, first formulation involves aggregating all objectives into one single objective, while the second formulation involves aggregating different robustness objectives into one, and finally the third formulation involving simultaneous optimization for all objectives with no aggregation. The optimization technique proposed in this paper is inspired by Population Based Incremental Learning [1], as it employs probabilistic modeling of promising solutions in order to guide the search for optimal solutions based on the optimization population.

The rest of the paper is organized as follows. Section II provides a background on intelligent watermarking and population based incremental learning, and illustrates the proposed multi-objective intelligent watermarking system using PBIL. Section III describes the experimental methodology used for computer simulations, and finally Section IV and Section V list experimental results along with analysis, and the conclusion of this paper respectively.

\section{INTELLIGENT WATERMARKING WITH Multi-ObJective Population Based InCREMENTAL LEARNING}

Intelligent watermarking [8] was introduced to resolve the trade-off between quality and robustness of the watermarking system using non-conventional methods as Evolutionary Computating (EC) methods like Genetic Algorithms and Partcile Swarm Optimization. These have proved efficiency in resolving such optimization problems. The optimization problem seeks to optimize the fitness for both quality represented by Peak Signal to Noise Ratio (PSNR) and robustness represented by Normalized Correlation (NC) [7].

Many authors have proposed aggregating both quality and robustness fitness into one objective for simplicity utilizing different aggregation weights. Few authors have proposed multi-objective formulation [6], [5] as it corresponds to the trade-off among multiple robustness objectives for different types of manipulations and quality objectives. However, Multiobjective formulation provides means for simultaneous optimization of different objectives of different scales without favoring one objective over the others.

Population Based Incremental Learning method proposed by Baluja in 1994 [1] is developed by combining GA and competitive learning to reduce the difficulties on the crossover and mutation operations in a GA, while retaining the stochastic search nature of the GA. PBIL therefore is similar to GA in using a binary encoded representation. The salient feature of PBIL is introducing real valued probability vector. The value of each element of this vector is the probability of having a 1 in that particular bit position of the encoded chromosome. In every generation, this probability vector is used to generate a new population in such a way that the probability for the $i$ th bit of a chromosome to become 1 is proportional to the value of the $i$ th element of this probability vector. After evaluating the objective functions of the new population, this probability vector is updated by using only the best individual of the

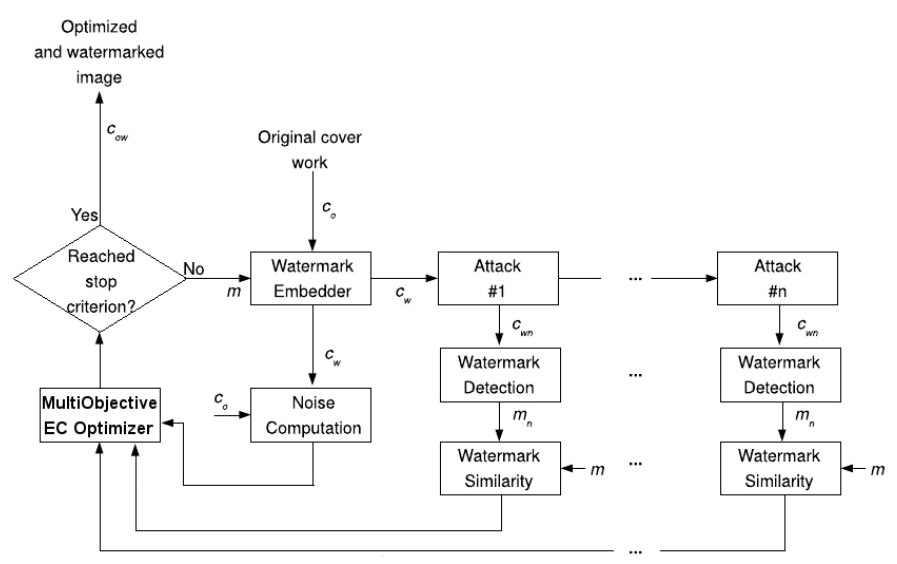

Fig. 1. Block diagram for an intelligent watermarking system with an EC multi-objective optimization component based on PBIL [8].

current population to help it shifting towards the chromosome of this new individual.

Bureerat and Sriworamas [2] proposed changes to PBIL algorithm to handle multi-objective optimization problems. In this algorithm the probability vector is replaced with probability matrix, where each row in this matrix represents the probability vector to create sub-population of individuals. An external archive is proposed to be used to store the nondominated solutions found throughout iterations.

The multi-objective PBIL module shown in Figure 1 is proposed for intelligent watermarking where objectives of quality and robustness against different attacks are optimized simultaneously. This algorithm provides multiple non-dominated solutions (Pareto front), which allows the system operator to tune the watermarking system's quality and robustness. This is accomplished by choosing the best solution with regards to robustness against certain attack to be the optimal embedding for digitized document images. The Multi-objective PBIL optimizer used is detailed in Algorithm 1.

The watermark embedding/extracting algorithm used in the proposed system is an algorithm proposed by Shieh et al [7] where the original host image is not required in watermark extraction phase. This algorithm is based on transforming the host image into DCT domain and divide it into $8 \times 8$ pixels image blocks, and the watermark bits are distributed among these image blocks. The changes due to embedding the watermark is dependent on the image content to minimize the visual impact measured by PSNR to represent quality fitness. Then watermarking attacks are applied to the watermarked image, and the watermark is extracted from the attacked watermarked image, the correlation between the extracted watermark and the original watermark is measured using $\mathrm{NC}$ representing the robustness of the watermark against the attack. The main objective of the proposed intelligent watermarking system is to maximize both PSNR and different NC for different attacks using incremental learning techniques. 


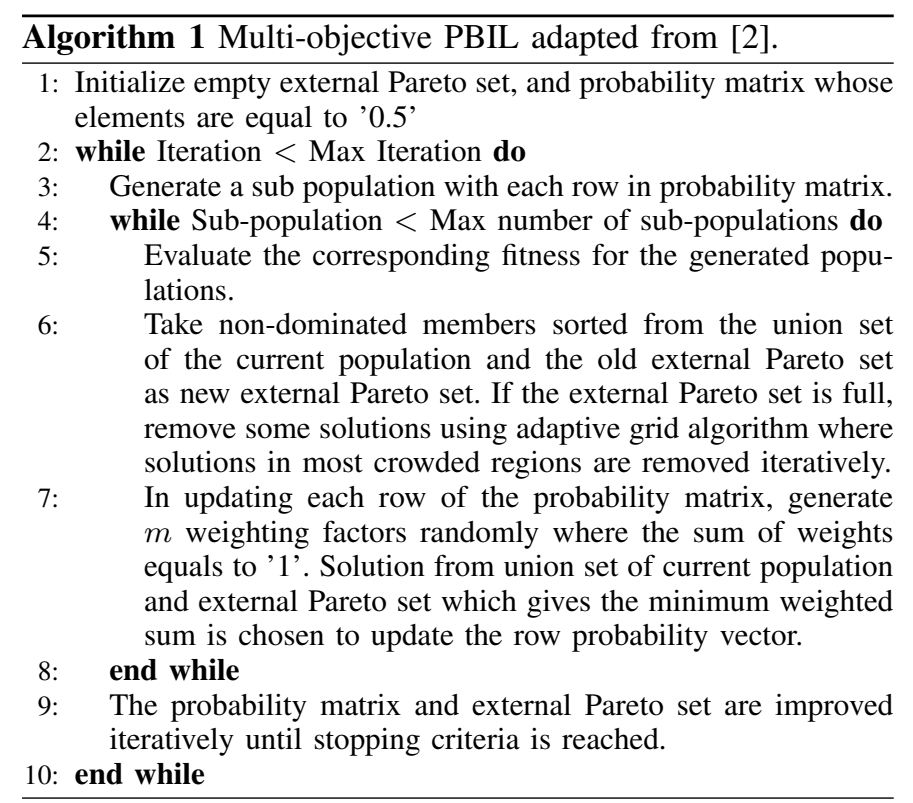

\section{EXPERIMENTAL METHODOLOGY}

The attacks considered in experiments are Gaussian Low Pass Filter (LPF) with standard deviation 0.9 using $3 \times 3$ pixel blocks, JPEG compression with quality factor $=80$, and Median Filter (MF) with $3 \times 3$ pixel blocks. Binary representation was used to encode individuals, where each individual have binary representation of frequency bands in binary encoding per image $8 \times 8$ block multiplied by number of blocks in the host image. Each frequency band is represented by 6-bits to identify the $\mathrm{AC}$ frequency coefficient $(0-63)$ to embed the watermark in.

In the experiments, a $128 \times 128$ binary watermark image is embedded in a $512 \times 512$ grayscale host images using the system described in Figure 1. The capacity of watermark embedding can be calculated by dividing watermark number of bits 16,384 by the number of host image $8 \times 8$ blocks 4096 yielding 4 watermark bits to be embedded in each image block. The host image is divided into 4096 8x8 blocks, leading to an individual dimension equal to the product of the number of blocks (4096), number of embedding frequency bands per block (4), and number of bits for frequency band representation (6), which yields to dimensions equal to 98,304 binary bits. Optimization is performed using single objective GA, two objective optimization using multi-objective PBIL aggregating different robustness objectives into one single objective, and finally four objective optimization using multiobjective PBIL. All optimization were performed using population of 24 individuals for 200 iterations.

\section{RESUlts AND ANALYSIS}

Figure 2 shows the Pareto front produced when aggregating different robustness objectives into one objective optimized simultaneously with quality. Table I lists the non-dominated solutions in the Pareto front produced in this case. The results
TABLE I

PARETO FRONT PRODUCED FOR PBIL MULTI-OBJECTIVE EMBEDdiNG Rose $128 \times 128$ BINARY IMAGE IN LENA 512X512 GRAYSCALE IMAGE WITH AGGREGATION WEIGHT 10 FOR ROBUSTNESS OBJECTIVES.

\begin{tabular}{|l|l|l|l|l|}
\hline Soln & PSNR & NC1(LPF) & NC2(MF) & NC3(JPEG) \\
\hline $\mathbf{1}$ & 73.90 & 0.610 & 0.545 & 0.977 \\
\hline $\mathbf{2}$ & 66.77 & 0.612 & 0.544 & $\mathbf{0 . 9 8 3}$ \\
\hline $\mathbf{3}$ & 68.82 & 0.608 & $\mathbf{0 . 5 5 9}$ & 0.972 \\
\hline $\mathbf{4}$ & 62.21 & $\mathbf{0 . 6 1 6}$ & 0.554 & 0.976 \\
\hline $\mathbf{5}$ & $\mathbf{9 6 . 7 1}$ & 0.605 & 0.536 & 0.968 \\
\hline $\mathbf{6}$ & 71.14 & 0.613 & 0.545 & 0.980 \\
\hline $\mathbf{7}$ & 90.22 & 0.603 & 0.536 & 0.980 \\
\hline $\mathbf{8}$ & 78.24 & 0.610 & 0.541 & 0.980 \\
\hline $\mathbf{9}$ & 84.89 & 0.605 & 0.539 & 0.981 \\
\hline $\mathbf{1 0}$ & 81.56 & 0.604 & 0.541 & 0.980 \\
\hline $\mathbf{1 1}$ & 64.37 & 0.614 & 0.548 & 0.981 \\
\hline $\mathbf{1 2}$ & 85.00 & 0.605 & 0.539 & 0.980 \\
\hline
\end{tabular}

show best fitness for quality is the $5^{\text {th }}$ solution, for robustness against LPF is the $4^{\text {th }}$ solution, for robustness against MF is the $3^{\text {rd }}$ solution, and finally for the robustness against JPEG compression is the $2^{\text {nd }}$ solution. This demonstrates the main advantage for using multi-objective formulation. A system operator can alternate his choice for the most suitable solution with respect to specific attack for different types of digitized documents without resulting to the expensive reoptimization proces. For example, the operator can choose the second non-dominated solution if the digitized documents need higher robustness against JPEG compression, specially if these documents will be distributed on mobile devices with possible compression performed on the digitized documents. Alternatively, the operator can choose the fifth solution to get the minimum visual impact on the watermarked image if the watermarked digitized documents requires further processing like extracting features from these watermarked digitized documents.

Table II shows the fitness produced using different formulations for the MOOP. Comparing to results in [7] is hard because tunings of considered attacks might be different. However the first experiment optimizing bands using single objective GA represents the same case with the same tunings of attacks used for different variants of multi-objective optimization experiments. For all non-dominated solutions in the produced Pareto front, best fitness achieved with respect to each objective is added for multi-objective formulation results.

The results show faster convergence for PBIL with a two objective optimization compared to GA single objective optimization. Here, convergence is reached with less iterations utilizing the experience of previous generations using incremental learning concepts. The best fitness achieved by two objective optimization is nearly equal fitness for PSNR and NC1 (LPF), and better fitness for NC2 (MF) and NC3 (JPEG). In the case 
TABLE II

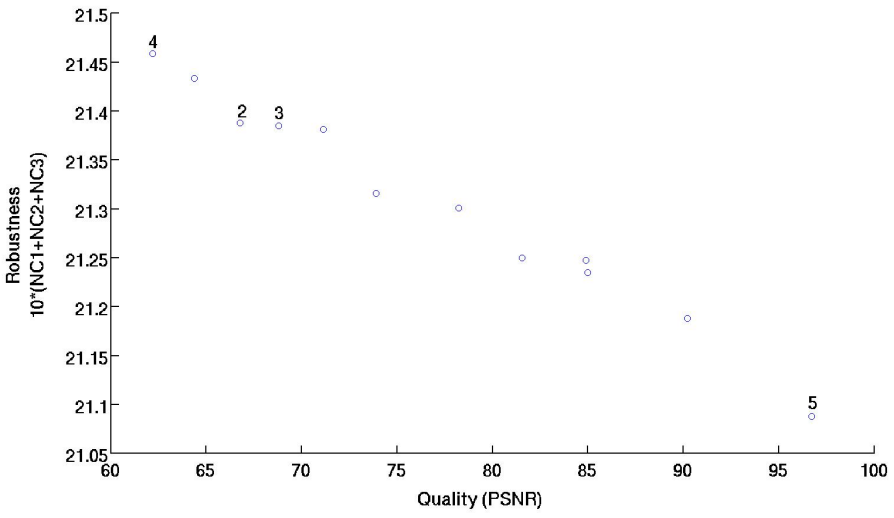

Fig. 2. Pareto front produced for PBIL multi-objective embedding Rose $128 \times 128$ binary image in Lena $512 \times 512$ grayscale image with aggregation weight 10 for robustness objectives. The best solutions 2,3,4, and 5 from Table I are enumerated

of a four objective optimization, aggregation is avoided totally in optimization process, and the fitness achieved without aggregation has better PSNR, NC2 (MF), and NC3 (JPEG), and the nearly same fitness for $\mathrm{NC1}(\mathrm{LPF})$. The four objective optimization formulation yields the best fitness compared to other formulations, although the convergence is reached with more iterations.

As for robustness against more attacks, it is computationally complex to formulate the optimization problem for tens of objectives. It is proposed to group related attacks into single objective to reduce the complexity of the optimization problem. The classification of watermarking attacks proposed by Voloshynovskiy et al [9] could be utilized to group attacks according to their nature. Another alternative is to use coevolution optimization techniques using multiple sub-populations handling the multiple objectives like VEPSO method [3].

\section{Conclusion}

This paper proposes a multi-objective PBIL module for intelligent watermarking system for grayscale images, and experiments prove the efficiency of this module and the formulation as multi-objective optimization. The proposed module provides means for watermarking operators to tune their systems to be more robust against specific attacks for set of digitized document images, without the need to repeat the expensive optimization problem after changing priority of robustness objectives. Different optimization problem formulation are evaluated and compared in this paper with respect to the best fitness for all objectives including quality and robustness against different attacks. Also the impact of using incremental learning technique on the number of iterations to reach convergence is studied in the experiments, and simulations prove reaching convergence in less iterations.
OPTIMIZATION RESULTS FOR EMBEDDING ROSE 128X128 BINARY IMAGE IN LENA 512X512 GRAYSCALE IMAGE

\begin{tabular}{|l|l|l|l|}
\hline & \multicolumn{3}{|c|}{ Fitness (PSNR+10(NC1+NC2+NC3)) } \\
\hline & GA & MO-PBIL & MO-PBIL \\
Iteration & 1-Objective & 2-Objective & 4-Objective \\
\hline $\mathbf{1}$ & 85.87 & 91.71 & 87.7 \\
\hline $\mathbf{5 0}$ & 115.2 & 117.8 & 109.4 \\
\hline $\mathbf{1 0 0}$ & 115.2 & 117.8 & 117.5 \\
\hline $\mathbf{1 5 0}$ & 118.5 & 117.8 & 117.5 \\
\hline $\mathbf{2 0 0}$ & 118.5 & 117.8 & 124.6 \\
\hline & \multicolumn{3}{|c|}{ Best Final Fitness } \\
\hline PSNR & 97.07 & 96.71 & 103.0 \\
\hline NC1(LPF) & 0.617 & 0.616 & 0.617 \\
\hline NC2(MF) & 0.555 & 0.559 & 0.560 \\
\hline NC3(JPEG) & 0.976 & 0.983 & 0.986 \\
\hline
\end{tabular}

\section{ACKNOWLEDGMENT}

This work was supported by the Natural Sciences and Engineering Research Council of Canada and BancTec Inc.

\section{REFERENCES}

[1] Shumeet Baluja. Population-based incremental learning: a method for integrating genetic search based function optimization and competitive learning. Technical report, CMU, 1994.

[2] Sujin Bureerat and Krit Sriworamas. Population-based incremental learning for multiobjective optimization. Soft Computing in Industrial Applications, 39:223-232, 2007.

[3] Marde Greeff and Andries P. Engelbrecht. Multi-Objective Swarm Intelligent Systems, chapter Dynamic Multi-objective Optimization Using PSO, pages 105-123. Springer-Verlag, 2010.

[4] Adil Haouzia and Rita Noumeir. Methods for image authentication: a survey. MultiMed Tools Appl, 39:1-46, 2008.

[5] D. Sal, M. Grana, and A. d Anjou. A moga to place the watermark in an hyperspectral image. In International Geoscience and Remote Sensing Symposium, pages 774-777, Denver, USA, August 2006.

[6] D. Sal Diaz and M. Grana Romay. Introducing a watermarking with a multi-objective genetic algorithm. (GECCO), 2005.

[7] Chin-Shiuh Shieh, Hsiang-Chen Huang, Feng-Hsing Wang, and JengShyang Pan. Genetic watermarking based on transform-domain techniques. Pattern Recognition, 37:555-565, 2004.

[8] Vellasques E., Granger E., and Sabourin R. Intelligent Digital Watermarking of Document Images, in Handbook of Pattern Recognition and Computer Vision, ed., C.H. Chen, 4th edition, 2010, pages 687-724.

[9] Sviatolsav Voloshynovskiy, Shelby Pereira, Thierry Pun, Joachim Eggers, and Jonathan K. Su. Attacks on digital watermarks: Classification, estimation-based attacks, and benchmarks. IEEE Communications Magazine, pages 118-126, 2001. 